\title{
Fractured Metal Wire in the Right Ventricle, a Cause of Cardiac Tamponade
}

\section{Mohamed Shafik Hashim, Dennis Elchanon Feierman}

Department of Anesthesiology, Maimonides Medical Center, Brooklyn, USA.

Email: dfeierman@maimoidesmed.org

Received November $11^{\text {th }}, 2011$; revised November $25^{\text {th }}, 2011$; accepted December $7^{\text {th }}, 2011$

\begin{abstract}
Cardiac tamponade is a surgical emergency. This case report illustrates a very peculiar situation, where the patient's condition deteriorated rapidly after arrival to the emergency room with a diagnosis of cardiac tamponade. Patient was promptly taken to the operating room and after evacuation of the pericardial effusion, had to be placed on cardio-pulmonary bypass in order to find and treat the source of the bleeding. A wire fragment was recovered from the right ventricle. This was identified as a broken piece of a guide wire used in placing a central venous catheter several years earlier.
\end{abstract}

Keywords: Tamponade; Complications; Central Venous Catheter

\section{Introduction}

The purpose of this case report is to increase awareness with respect to the serious delayed complication of guide wire sheering. This necessitates diligence in the placing of central venous lines (CVL) using the Seldinger technique; furthermore, sensitive studies need to be performed, if sheering has occurred, to find small guide wire fragments.

Recent open-heart surgery, invasive cardiac procedures, dissecting aortic aneurysm, anticoagulant overdose, cardiac trauma, viral infections, and acute myocardial infraction are some situations that can lead to cardiac tamponade. We describe a case of acute pericardial effusion caused by a metal-wire fragment embedded in the right ventricle (RV) for several years that subsequently perforated the myocardium.

\section{Case Report}

A 53-year-old obese male presented to the emergency room with acute onset of chest pain and dyspnea. Shortly after admission, his medical status rapidly deteriorated. He became hypotensive and required emergency tracheal intubation and intravenous infusion of vasopressors. His past medical history included hypertension, diabetes mellitus and gout. His past surgical history was signifycant for a motor vehicle accident over ten years ago, where he sustained multiple bone fractures that required a few months of hospitalization and was complicated by lower extremities deep vein thrombosis followed by in- sertion of inferior vena cava (IVC) filter. Additionally, he had a gastric bypass procedure seven years ago. In both hospitalizations he had multiple CVL insertions.

Laboratory blood tests were normal except for elevated white blood count $(14,000 / \mathrm{mL})$. An electrocardiogram showed a sinus tachycardia at rate of $144 \mathrm{bpm}$ with Q-wave in leads III and aVF. The chest x-ray film was unrevealing, in that it did not change the differential diagnosis. A bedside transthoracic echocardiogram (TTE) revealed a moderate-sized pericardial effusion with cardiac tamponade and right ventricular collapse. Cardiothoracic surgical service was consulted and the patient was brought to the operating room for emergency mediastinal exploration with a preoperative diagnosis of a dissecting ascending thoracic aortic aneurysm as a cause of the cardiac tamponade. The unstable hemodynamic status of the patient did not permit any further preoperative testing.

After establishing a left radial arterial line and intravenous induction of anesthesia, transesophageal echocardiogram (TEE) probe was inserted and showed moderate pericardial effusion with restricted left ventricle relaxation and right ventricle collapse (Figure 1), there was no visible ascending or descending aortic dissection. Despite the exclusion of the dissecting aortic aneurysm on the TEE, and due to the unexplained etiology of the pericardial effusion, the surgeon elected to cannulate the femoral artery and vein with the cardiopulmonary bypass machine on standby. A median sternotomy was performed and about $400 \mathrm{ml}$ of dark-colored blood was evacuated 
from the pericardium (it should be noted that if this was a slowly accumulating effusion, $400 \mathrm{ml}$ surrounding the heart would probable not have caused tamponade). Immediately, the patient's vital signs improved but the source of bleeding could not be readily identified. An additional epi-aortic echo did not show an aortic dissection or any other pathology. Despite the improvement in hemodynamics, a complete inspection of the posterior wall of the beating heart was not possible due to severe cardiovascular instability when lifting the heart and further bleeding; therefore, the cardio-pulmonary bypass was initiated. Lifting up the heart revealed a metal wire fragment (Figure 2) protruding from the postero-apical aspect of the right ventricle. It was removed and the myocardial puncture site was repaired. The patient was weaned off bypass uneventfully. He was discharged home 5 days later with no subsequent sequelae. The wire fragment was sent to pathology lab where it was examined and found to be a $1.7 \mathrm{~cm}$ long 28-gauge wire.

\section{Discussion}

Cardiac tamponade can result from various etiologies: acute external trauma to the heart, coagulation therapy, dissecting aortic aneurysm, pericardial infection, myocardial infarction or renal failure. However, in this case, the injury was internal from a fractured metal wire that had been lurking in the right ventricle for many years.

Usually the surgical approach to relieve the pericardial effusion is via a small sub-xiphoid incision except for cases of penetrating cardiac injury or dissecting aortic aneurysm where the surgical team seeks a wide open field to easily access the heart and the great vessels. In this case, the patient underwent an emergency midline sternotomy, initially, due to the suspicion of dissecting aortic aneurysm and obscure history of the causes leading to his pericardial effusion. A sub-xiphoid incision would have, temporarily, relieved the threatening hemodynamic compromise, but would have missed the diagnosis of the internal penetrating injury to the right ventricle and necessitated a subsequent more serious operation. In retrospect, the sternotomy was the appropriate approach. It was therefore possible to initiate cardiac bypass, which led us to locate the foreign body and prevent further bleeding into the pericardial space.

In the United State, physicians insert over 5 million CVL every year with $15 \%$ incidence of adverse effect [1]. This patient had multiple orthopedic operations, an inferior vena cava (IVC) filter placement and gastric bypass surgery in the past with several CVL insertions. There have been few reports in the literature of migrating fragments of IVC filters [2,3], surgical clips and Kirschner wires to the heart $[3,4]$. There has been a case report of a fractured recoverable inferior vena cava filter that caused cardiac tamponade [4]. This fragment was confirmed in the pathology report to be part of the guide wire, most likely from the one used during central venous line insertion more than seven years ago. A review of chest $\mathrm{x}$ ray of this patient taken 7 years before did not reveal any foreign body in the heart.

A guide wire often consists of a stainless steel core wound lengthways by a spring made of the same material (Figure 3). There has been a proposed guide wire design [5] which consists of thin wires of same material plied in a straight line to form a single guide wire (multiply).

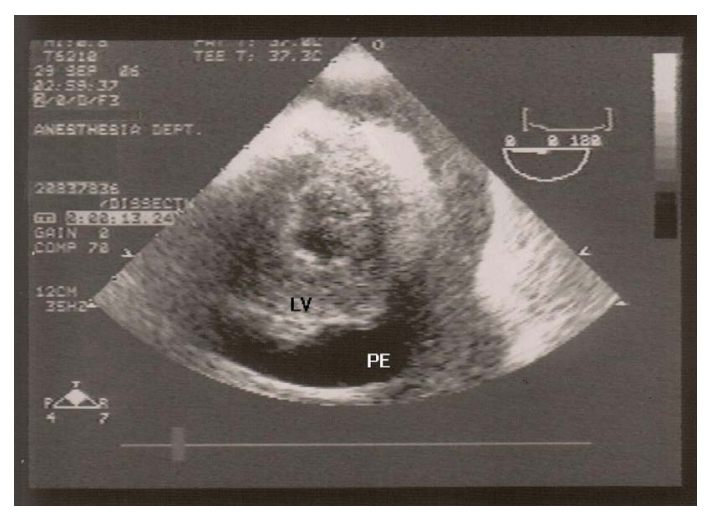

Figure 1. Post-induction transesophageal echocardiogram showing the left ventricle (LV) and the pericardial effusion (PE).

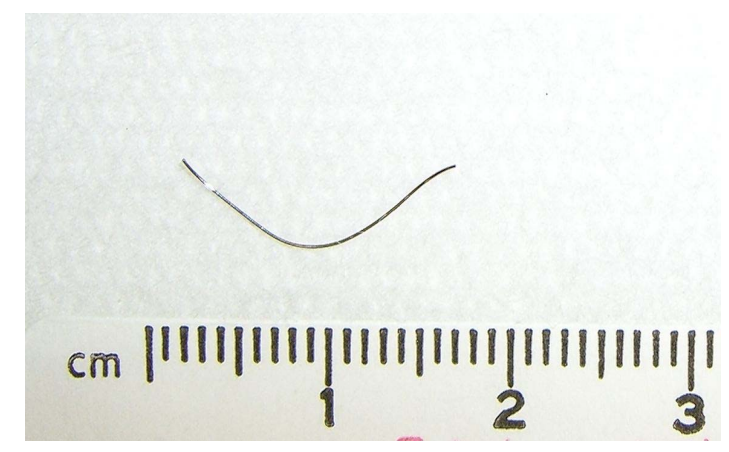

Figure 2. The removed metal-wire fragment.

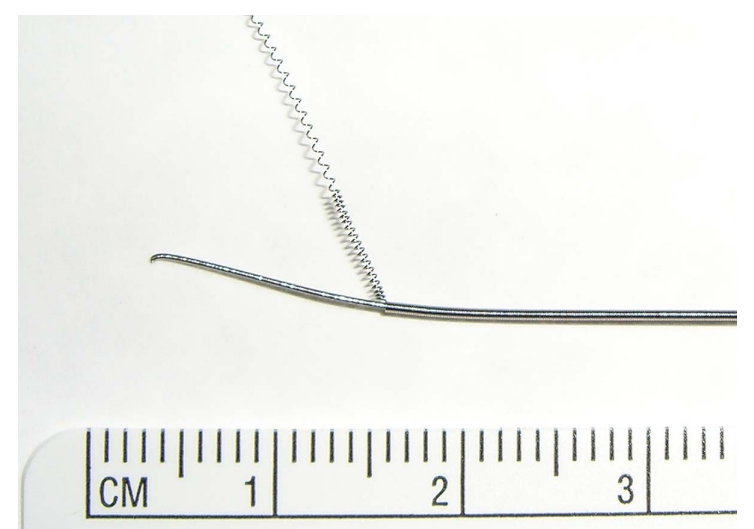

Figure 3. Conventional guide wire illustrating the spring layer and the metallic wire core. 
In our case, we think the guide wire fragment may have resulted from a phenomenon called "locking" $[5,6]$. This problem is thought to occur, during the surgical insertion, when the guide wire becomes bent and catches on the beveled part of the introducer needle [5,6]. The guide wire can not be advanced nor withdrawn. Forcefully withdrawing the guide wire while in this locked state may cause the spring coat of the guide wire, along with the metal core to break and further travel in the blood stream. It is therefore recommended that if locking occurs, both the metal needle and the guide wire be removed together as a unit. If the guide wire and introducer needle where remove simultaneously, it is unlikely that the guide wire would shear. Therefore, we make the hypothesis that the removal (or attempted removal) of the guidewire while in 'locked' position may have sheared off part of the guide wire structure (in this case the core), which then migrated to the heart where it became lodge in, and eventually perforated through the myocardium, resulting in the pericardial effusion and cardiac tamponade. This case serves as a reminder that great care must be taken during placement of central venous catheters using a guide wires through a metal needle, as fragments can rarely break-off and migrate to the heart, and even more rarely (as in this case) manifest as a life-threatening emergency.

Another plausible explanation is that the guide wire which is introduced from the superior vena cava, through the right atrium (RA) got entrapped with the IVC filter, which occurs as a result of over advancement of the guide wire. Therefore, it is recommended not to advance the guide wire beyond $20 \mathrm{~cm}$. Forceful removal can be catastrophic and the recommendation is to consult an interventional radiology or vascular team in order to free the guide wire under imaging. It may be that the guide wire fragment had been stuck for years against the IVC filter and eventually migrated to the RA, RV and caused a myocardial perforation. Another explanation is the wire immediately migrated to the heart and it took years for it to penetrate through and puncture the RV.

There are a handful of cases in literature where foreign objects from the heart have been removed percutaneously $[6,7]$ or surgically, but the migrating bodies were detected within days to weeks. This is the only case, to our knowledge, where an inner piece of the guide wire has fragmented and then embedded in the right ventricle for several years to manifest later as life-threatening cardiac tamponade requiring cardio-pulmonary bypass for removal.
Thus, to avoid similar complication, we recommend that physicians refrain from advancing the guide wire more than $20 \mathrm{~cm}$, and always visually inspect the tip of the guide wire after withdrawal. Another way to avoid shearing is to use the plastic angio-catheter instead of the metal needle. Additionally, we recommend close supervision of trainees during line insertion. In any case, if resistance is met during withdrawal, the guide wire and the introducing needle should be removed simultaneously without force. If a fragment of the guide wire tip is missing, the vascular or interventional radiology team must be consulted, although it is hard to detect a fine piece of wire on X-ray. Finally, the most important practice is to inform the patient about any adverse event during the CVL insertion.

\section{REFERENCES}

[1] D. C. McGee and M. K. Gould, "Preventing Complications of Central Venous Catheterization,” New England Journal of Medicine, Vol. 348, No. 12, 2003, pp. 11231133. doi:10.1056/NEJMra011883

[2] F. Castaneda, M. Herrera, A. H. Cragg, E Salamonowitz, G. Lund, W. R. Castaneda-Zuniga and K. Aplatz, "Migration of a Kimray-Greenfield Filter to the Right Ventricle,” Radiology, Vol. 149, No. 3, 1983, p. 690.

[3] G. M. Actia Dato, A. Arslanian, P. Di Marzio, P. L. Filosso and E. Ruffini, "Posttraumatic and Iatrogenic Foreign Bodies in the Heart: Report of Fourteen Cases and Review of the Literature," Journal of Thoracic and Cardiovascular Surgery, Vol. 126, No. 2, 2003, pp. 408-414. doi:10.1016/S0022-5223(03)00399-4

[4] I, Saeed, M, Garcia and K. McNicholas, "Right Ventricular Migration of a Recovery IVC Filter's Fractured Wire with Subsequent Pericardial Tamponade," CardioVascular and Intervention al Radiology, Vol. 29, No. 4, 2006, pp. 685-686. doi:10.1007/s00270-005-0136-7

[5] T. Suzuki, K. Ito, J. Nishiyama, K. Hasegawa, M. Kanazawa and H. Fukuyama, "Development of a Safe Guide wire,” Journal of Anesthesia, Vol. 20, No. 1, 2006, pp. 64-67. doi:10.1007/s00540-005-0361-X

[6] H, Arjomand, S, Surabhi and N. M. Wolf, "Right Ventricular Foreign Body: Percutaneous Transvenous Retrieval of a Greenfield Filter from the Right Ventricle-A Case Report,” Angiology, Vol. 54, No. 1, 2003, pp. 109113. doi:10.1177/000331970305400114

[7] P. Rasuli, R. Mehran, G. French, M. Turek, K. A. Lalonde and P. Cardinal, "Percutaneous Retrieval of a Vena Cava Filter from the Right Atrium: Case Report," Canadian Association of Radiologists Journal, Vol. 51, No. 1, 2000, pp. 30-35. 